\title{
Investigation on the Competition Interaction of Synthetic Food Colorants and Ciprofloxacin Hydrochloride with Bovine Serum Albumin by Fluorescence Spectroscopy
}

\author{
Baosheng Liu, Chao Yang, Jing Wang, Chunli Xue, and Yunkai Lü \\ Key Laboratory of Medical Chemistry and Molecular Diagnosis, Ministry of Education, College of Chemistry \& Environmental Science, \\ Hebei University, Baoding 071002, China \\ Correspondence should be addressed to Baosheng Liu, lbs@hbu.edu.cn
}

Received 12 June 2011; Accepted 3 September 2011

Academic Editor: Ali-Akbar Saboury

Copyright () 2011 Baosheng Liu et al. This is an open access article distributed under the Creative Commons Attribution License, which permits unrestricted use, distribution, and reproduction in any medium, provided the original work is properly cited.

\begin{abstract}
The effects of synthetic food colorants like tartrazine (TTZ), sunset yellow (SY), and erythrosine (ETS) on the binding reaction between ciprofloxacin hydrochloride (CPFX) and bovine serum albumin (BSA) were investigated by fluorescence spectroscopy in the aqueous solution of $\mathrm{pH}=7.40$. Results showed that $\mathrm{CPFX}$ caused the fluorescence quenching of BSA through a static quenching procedure and the primary binding site was located at subdomain IIA of BSA (site I). According to the calculated thermodynamic parameters, it confirmed that CPFX bound to BSA by electrostatic interaction. In addition, the colorants affected the formation of BSA-CPFX complex. This resulted in an increase of the free, biological active fraction of CPFX. The binding distance of BSA-CPFX systems was evaluated according to Förster's theory. Results suggested that the binding distance were increased in the presence of synthetic food colorants.
\end{abstract}

\section{Introduction}

When drugs are absorbed, they enter the circulatory system and bind to serum albumin extensively and reversibly [1]. The effectiveness of drugs depends on their combination abilities. Binding of drugs to serum albumin has significance in pharmacology, since it controls their free concentrations and, as a consequence to the degree and time of action in the body, affects duration and intensity of their effects [2-4]. In other words, binding to serum albumin will significantly affect the distribution, metabolism, and excretion of drugs. Drugs are subjected to the influences of many factors in vivo, among which dietary habits cannot be ignored. Interactions between diet and drugs reflect the change of pharmacodynamics and the incompatibilities. There are various researches about the influences of sugar, wine, and tea on the efficacy of a medicine $[5,6]$. However, researches about the effects of synthetic food colorants on medication have not been reported.

Ciprofloxacin hydrochloride (CPFX, the structure is shown in Figure 1) which belongs to the third-generation synthetics of quinolones shows striking potency against enteric Gram-negative bacilli, lesser activity against nonenteric Gram-negative bacilli and staphylococci, and generally marginal activity against streptococci and anaerobes $[7,8]$. It has received much attention because of its broad-spectrum pharmacological activities and extensive biological effects. Tartrazine (TTZ), sunset yellow (SY), and erythrosine (ETS, structures are shown in Figure 1) are common synthetic food colorants, which are widely used in food like drinks, candies, and in sugar-coated capsules for pharmaceutical preparations [9]. The effects of three synthetic food colorants TTZ, SY, and ETS on the CPFX-BSA complex were investigated by fluorescence spectroscopy in this paper. Results showed that these colorants affected the formation of CPFX-BSA complex and further affected the efficacy of the medicine. The research was of a certain theoretical significance for patients' reasonable diet and clinical medication.

\section{Experimental}

2.1. Apparatus. All fluorescence spectra were recorded with a Shimadzu RF-540 spectrofluorophotometer and a Hitachi 
<smiles>O=C(O)c1cn(C2CC2)c2cc(N3CCNCC3)c(F)cc2c1=O</smiles>

(a)

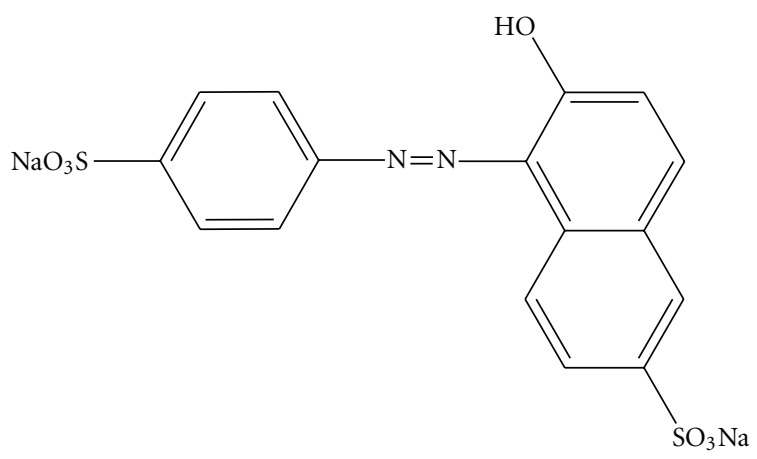

(c)

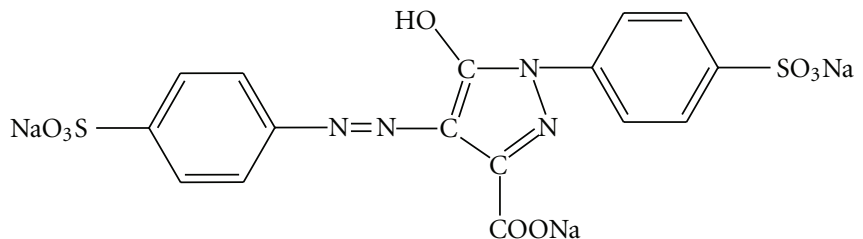

(b)<smiles>[N+]OC1C(I)=Cc2c(oc3c(I)c(=O)c(I)cc-3c2-c2ccccc2C(N)=O)C1I</smiles>

(d)

Figure 1: Molecular structures of ciprofloxacin hydrochloride (a), tartrazine (b), sunset yellow (c), and erythrosine (d).

F-4500 spectrofluorophotometer. Absorption was measured with a UV-vis recording spectrophotometer (UV-265 Shimadzu, Japan). All pH measurements were made with a pHS-3C precision acidity meter (Leici, Shanghai). All temperatures were controlled by CS501 superheated water bath (Nantong Science Instrument Factory).

2.2. Materials. Ciprofloxacin hydrochloride (CPFX) was obtained from Monitor of Chinese Veterinary Medicine (no less than $99.9 \%$ pure). Stock solutions $(1.0 \mathrm{mM})$ of CPFX were prepared by dissolving the drug in water. Bovine serum albumin (BSA, 10.0 $\mu \mathrm{M}$ ) was purchased from Sigma Company (no less than 99\% pure). Tartrazine (TTZ), sunset yellow (SY), and erythrosine (ETS) were purchased from Aldrich (Wisconsin, USA). Stock solutions $(0.1 \mathrm{mM})$ of them were prepared by dissolving the food colorants in water. Warfarin (WF), ibuprofen (IB), and digitoxin (DG) were all obtained from Chinese Institute of Drug and Biological Products. Tris- $\mathrm{HCl}(0.05 \mathrm{M})$ buffer solution containing $\mathrm{NaCl}$ $(0.2 \mathrm{M})$ was used to keep the $\mathrm{pH}$ of the solution at 7.40 . $\mathrm{NaCl}$ solution was used to maintain the ionic strength of the solution. All reagents were of analytical grade, and doubledistilled water was used throughout the experiments.

According to Steiner, if the absorption values are less than 0.3 , the fluorescence intensity is corrected for the absorption of excitation light and reabsorption of emitted light using the following relationship [10]:

$$
F_{\text {cor }}=F_{\text {obs }} \times e^{\left(A_{\mathrm{ex}}+A_{\mathrm{em}}\right) / 2}
$$

where $F_{\text {cor }}$ and $F_{\text {obs }}$ are the corrected and observed fluorescence intensities, respectively. $A_{\mathrm{ex}}$ and $A_{\mathrm{em}}$ are the absorbance values of the system at excitation and emission wavelengths, respectively. The fluorescence intensity used in this paper was corrected.

\subsection{Procedures}

2.3.1. Fluorescence Quenching of the BSA-CPFX Systems. In a typical fluorescence measurement, $1.0 \mathrm{~mL}$ of $\mathrm{pH}=7.40$ Tris- $\mathrm{HCl}, 1.0 \mathrm{~mL}$ of $10.0 \mu \mathrm{M}$ BSA solution, and $0.0,0.1$, $0.5,1.0,1.5,2.0,2.5,3.0,3.5$, and $4.0 \mathrm{~mL}$ of $0.1 \mathrm{mM}$ CPFX were added into a $10 \mathrm{~mL}$ colorimetric tube successively. The samples were diluted to scaled volume with water, mixed thoroughly by shaking, and kept static for $20 \mathrm{~min}$ at different temperatures (298 and $303 \mathrm{~K}$ ). The excitation and emission wavelengths for BSA were 280 and $342 \mathrm{~nm}$, respectively, with the excitation and emission slit widths set at $5 \mathrm{~nm}$. Meanwhile, we made use of the fluorescence intensity of BSA without any drugs as $F_{0}$ to study the quenching effects of CPFX to BSA.

2.3.2. Site Marker Competitive Experiments. The site marker WF, IB, or DG was, respectively, added to the mixture of the binary systems described above, and the ratio of each site marker and BSA was kept at 1 . The procedure was the same as that in Section 2.3.1 above. We made use of the fluorescence intensity of BSA only with site makers as $F_{0}$ to study the primary binding site for CPFX on BSA.

2.3.3. Effects of Synthetic Food Colorants on the Interaction between CPFX and BSA. Tris- $\mathrm{HCl}$, BSA (same as Section 2.3.1), $1.0 \mathrm{~mL}$ of $20.0 \mu \mathrm{M}$ TTZ (or SY, ETS) solution, 
and $0.0,0.1,0.5,1.0,1.5,2.0,2.5,3.0,3.5$, and $4.0 \mathrm{~mL}$ of $0.1 \mathrm{mM}$ CPFX were added into a $10 \mathrm{~mL}$ colorimetric tube successively. The procedure was the same as that in Section 2.3.1 above. Meanwhile, make use of the fluorescence intensity of BSA-food colorants without any drugs as $F_{0}$, to study the effects of synthetic food colorants on the interaction between CPFX and BSA. Then, fluorescence spectra were measured as previously described with different concentration of food colorants $(50.0 \mu \mathrm{M})$ to discuss the influences of their concentrations on drugs' binding.

\section{Results and Discussion}

3.1. Fluorescence Quenching of the Binary Systems. Proteins are considered to have intrinsic fluorescence due to the presence of amino acids, mainly tryptophan, tyrosine, and phenylalanine. Figure 2 showed that the emission spectra of CPFX and BSA were overlapped. The fluorescence difference spectra of BSA-CPFX system were obtained to eliminate the interference by subtracting the spectra of the CPFX in Figure 3(A) from that of the corresponding BSA-CPFX in Figure 2. The results were shown in Figure 3(B). It can be seen that the addition of CPFX caused a gradual decrease in the fluorescence emission intensity of BSA. The fluorescence intensities of BSA-CPFX system used in this paper were at $342 \mathrm{~nm}$ in the fluorescence difference spectra.

Fluorescence quenching can occur by different mechanisms; it may be dynamic quenching, resulting from the collisional encounter between the drug and protein, or static quenching, resulting from the formation of a ground-state complex between the drug and protein. Higher temperature would result in faster diffusion and typically the dissociation of weekly bound complexes, leading to larger amount of dynamic quenching and smaller amounts of static quenching, respectively [11]. In order to confirm the quenching mechanism, the fluorescence quenching data are analyzed by the Stern-Volmer equation [12]

$$
\frac{F_{0}}{F}=1+K_{q} \tau_{0}[Q]=1+K_{\text {sv }}[Q]
$$

where $F_{0}$ and $F$ are the fluorescence intensities in the absence and presence of quencher, respectively. $\tau_{0}$ is the average lifetime of fluorescence without quencher, which is about $10^{-8} \mathrm{~s}, K_{\mathrm{sv}}$ is the Stern-Volmer quenching constant, $K_{q}$ is the quenching rate constant of bio-molecule, and $[Q]$ is the concentration of the quencher. Based on the linear fit plot of $F_{0} / F$ versus $[Q]$, the $K_{q}$ value can be obtained. The calculated results are shown in Table 1. At different temperatures, the values of $K_{q}$ were much greater than the maximum diffusion collision quenching rate constant of various drugs with proteins $\left(2 \times 10^{10} \mathrm{M}^{-1} \mathrm{~s}^{-1}\right)$. This showed that the quenching was not initiated by dynamic collision but via formation of complexes. In addition, the values of $K_{q}$ decreased with the increase in temperature. This also suggested that the quenching was a static process.

When small molecules bind independently to a set of equivalent sites on a macromolecule, the binding constant

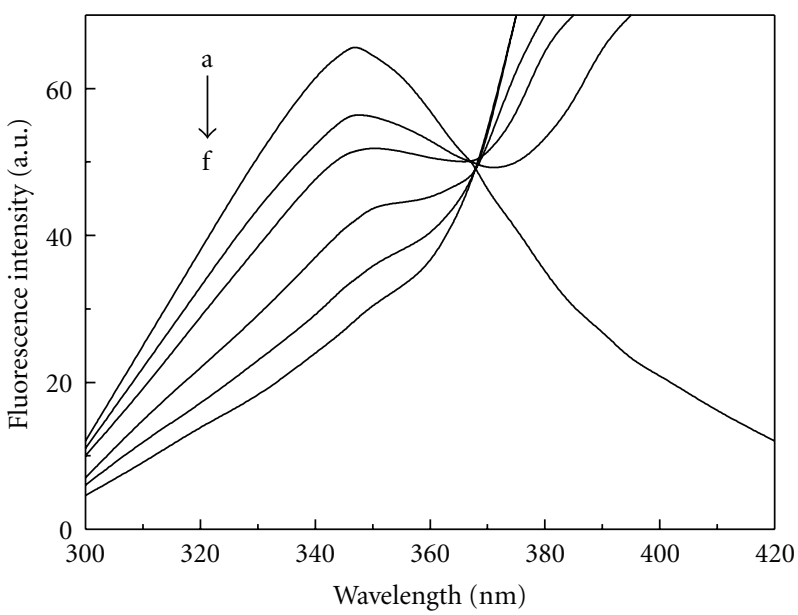

FIGURE 2: Quenching fluorescence spectra of BSA-CPFX systems $(T=298 \mathrm{~K}) . C_{\mathrm{BSA}}=1.0 \mu \mathrm{M}, \mathrm{a} \sim \mathrm{f}, C_{\mathrm{CPFX}}=0.0,5.0,10.0,20.0,30.0$, and $40.0 \mu \mathrm{M}$, respectively.

$\left(K_{a}\right)$ and the numbers of binding sites $(n)$ can be determined by $[13,14]$

$$
\lg \frac{F_{0}-F}{F}=n \lg K_{a}+n \lg \left(\left[Q_{t}\right]-n \frac{F_{0}-F}{F_{0}}\left[B_{t}\right]\right),
$$

where $\left[Q_{t}\right]$ and $\left[B_{t}\right]$ are the total concentration of quencher and protein, respectively. The curve of $\lg \left[\left(F_{0}-F\right) / F\right]$ versus $\lg \left\{\left[Q_{t}\right]-n\left[B_{t}\right]\left(F_{0}-F\right) / F_{0}\right\}$ is drawn and fitted linearly on the assumption that $n$ in the bracket is equal to 1 . The value of $n$ obtained from the slope of the plot is substituted into the bracket and the curve above is drawn again. In that way, the values of $n$ and $K_{a}$ can be obtained by iteration method. The corresponding calculated results were shown in Table 1. It shows that the value of $n$ was approximately equal to 1 , which indicated that there was one class of binding sites of CPFX on BSA. Meanwhile, the values of $K_{a}$ decreased with the increasing temperature, further suggested that the quenching was a static process for the dissociation of bound complexes.

3.2. Type of Interaction Force of the Binary Systems. The interaction forces between the small organic molecule and biological macromolecule include hydrophobic force, hydrogen bond, Van der Waals force, and electrostatic interactions. The signs and magnitudes of the thermodynamic parameters enthalpy change $(\Delta H)$ and entropy change $(\Delta S)$ can account for the main forces involved in the binding reaction. The reaction enthalpy change can be regarded as constant if the temperature changes little. Negative $\Delta H$ and positive $\Delta S$ indicate that electrostatic interaction plays a major role in the binding reaction. Positive $\Delta H$ and $\Delta S$ are generally considered as the evidence for typical hydrophobic interactions. In addition, Van der Waals force and hydrogen bonding formation in low dielectric media are characterized 

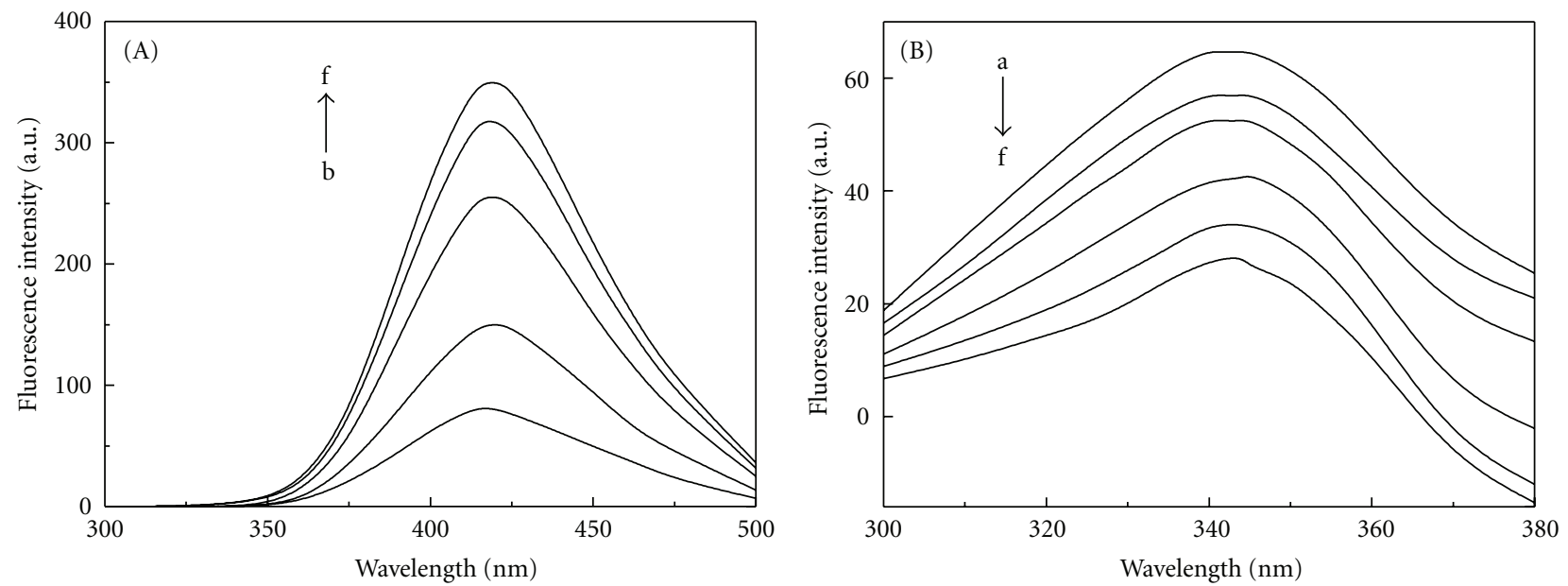

FIGURE 3: (A) Fluorescence spectra of CPFX; (B) Fluorescence difference spectra of BSA-CPFX system obtained by subtracting Figure 3(A) from Figure $2(T=298 \mathrm{~K}) . C_{\mathrm{BSA}}=1.0 \mu \mathrm{M}$ in all systems, $\mathrm{a} \sim \mathrm{f}, C_{\mathrm{CPFX}}=0.0,5.0,10.0,20.0,30.0$, and $40.0 \mu \mathrm{M}$, respectively.

TABLE 1: Quenching reactive parameters of BSA and CPFX.

\begin{tabular}{lcccr}
\hline$T /(\mathrm{K})$ & $K_{q} /\left(\mathrm{M}^{-1} \mathrm{~s}^{-1}\right)$ & $R^{\mathrm{a}}$ & $K_{a} /\left(\mathrm{M}^{-1}\right)$ & $n$ \\
\hline 298 & $3.13 \times 10^{12}$ & 0.9954 & $3.02 \times 10^{4}$ & 1.21 \\
303 & $2.96 \times 10^{12}$ & 0.9932 & $2.71 \times 10^{4}$ & 0.9991 \\
\hline
\end{tabular}

$R^{\mathrm{a}}$ is the linear relative coefficient of $F_{0} / F \sim[Q] ; R^{\mathrm{b}}$ is the linear relative coefficient of $\log \left(F_{0}-F\right) / F \sim \log \left\{\left[Q_{t}\right]-n\left[B_{t}\right]\left(F_{0}-F\right) / F_{0}\right\} . K_{q}$ is the quenching rate constant; $K_{a}$ is the binding constant; $n$ is the number of binding site.

by negative $\Delta H$ and $\Delta S$ [15]. The interaction parameters can be calculated on the basis of the van't Hoff equation [16]

$$
\begin{gathered}
\ln \frac{K_{2}}{K_{1}}=\left(\frac{1}{T_{1}}-\frac{1}{T_{2}}\right) \frac{\Delta H}{R}, \\
\ln K=-\frac{\Delta H}{R T}+\frac{\Delta S}{R} .
\end{gathered}
$$

The free energy change $\Delta G$ at different temperatures can be calculated from

$$
\Delta G=\Delta H-T \Delta S .
$$

According to the binding constants $K_{a}$ of CPFX to BSA at the two temperatures above, the thermodynamic parameters were obtained. Therefore, the values of $\Delta H^{\theta}$, $\Delta S^{\theta}$, and $\Delta G^{\theta}$ were $-16.22 \mathrm{~kJ} \mathrm{~mol}^{-1}, 31.32 \mathrm{~J} \mathrm{~mol}^{-1} \mathrm{~K}^{-1}$, and $-25.56 \mathrm{~kJ} \mathrm{~mol}^{-1}(T=298 \mathrm{~K})$, respectively. The negative value of $\Delta G$ clarified an automatic reaction between CPFX and BSA. The negative value of $\Delta H$ and positive value of $\Delta S$ showed that CPFX mainly bound to BSA by the electrostatic attraction.

3.3. Identification of the Binding Sites of CPFX on the BSA. Crystal structure analysis has revealed that the tertiary structure of serum albumin is composed by three domains: I, II, and III. Each domain is constituted by a cylinder formed by six helices, and each one of these domains is constituted by two subdomains formed by three helices that are covalently linked by their double Cys bridge [17]. The principal regions of ligand binding sites of albumin are located in hydrophobic cavities in subdomain IIA and IIIA [18]. There are three binding sites in albumin, namely, site I, II, and III. X-ray studies suggested that site I and II are located in subdomain IIA and IIIA of albumin, respectively [19]. Many ligands bind specifically to serum albumin, such as WF for site I, IB for site II, and DG for site III [20, 21]. In order to identify the binding location of CPFX on BSA, site marker competitive experiments were carried out using some drugs mentioned above.

The binding constants of systems in the presence of site marker (WF, IB, and DG, resp.) $K_{a}^{\prime}$ were obtained according to Section 2.3.2. The $\varphi_{1}$ represents the rate of change of binding constants for systems after adding site markers. It was determined using

$$
\varphi_{1}=\frac{\left(K_{a}^{\prime}-K_{a}\right)}{K_{a}},
$$

where $K_{a}$ is the binding constant of the BSA-CPFX systems in the absence of site markers. Table 2 gives the corresponding calculated results. The values of $\varphi_{1}$ were all negative, indicating the binding constants of the binary systems in the presence of site markers were decreased. The most descendent were the systems with adding WF, indicating the presence of competition for the same binding site of BSA when WF and CPFX coexist. The above experimental results demonstrated that the binding of CPFX to BSA was mainly located within site I (subdomain IIA).

3.4. Effects of Synthetic Food Colorants on the Binding Constants between CPFX and BSA. The fluorescence quenching 

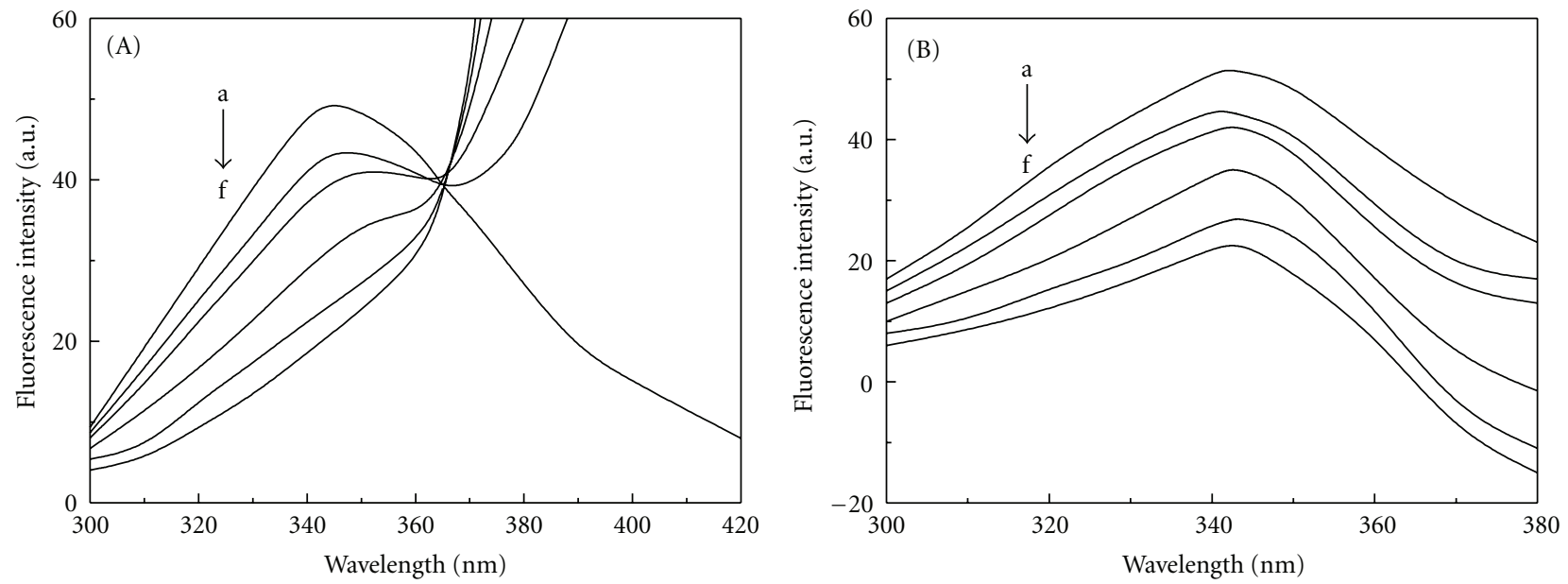

FIGURE 4: (A) Quenching fluorescence spectra of BSA-SY-CPFX system; (B) Fluorescence difference spectra of BSA-SY-CPFX system obtained by subtracting Figure $3(\mathrm{~A})$ from Figure $4(\mathrm{~A}) . C_{\mathrm{BSA}}=1.0 \mu \mathrm{M}, C_{\mathrm{SY}}=5.0 \mu \mathrm{M}, \mathrm{a} \sim \mathrm{f}, C_{\mathrm{CPFX}} /(\mu \mathrm{M}): 0.0,5.0,10.0,20.0,30.0$, and 40.0 , respectively, $T=298 \mathrm{~K}$.

TABLE 2: Binding constants $K_{a}^{\prime}$, number of binding sites $n$ and the rate of change of binding constants $\varphi_{1}$ for BSA-CPFX system with adding site makers reagents $(T=298 \mathrm{~K})$.

\begin{tabular}{lccc}
\hline System & $K_{a}^{\prime} /\left(\mathrm{M}^{-1}\right)$ & $n$ & $\varphi_{1} /(\%)$ \\
\hline BSA-WF-CPFX & $2.03 \times 10^{4}$ & 0.989 & -32.8 \\
BSA-IB-CPFX & $2.70 \times 10^{4}$ & 1.04 & -10.6 \\
BSA-DG-CPFX & $2.84 \times 10^{4}$ & 0.935 & -6.96 \\
\hline
\end{tabular}

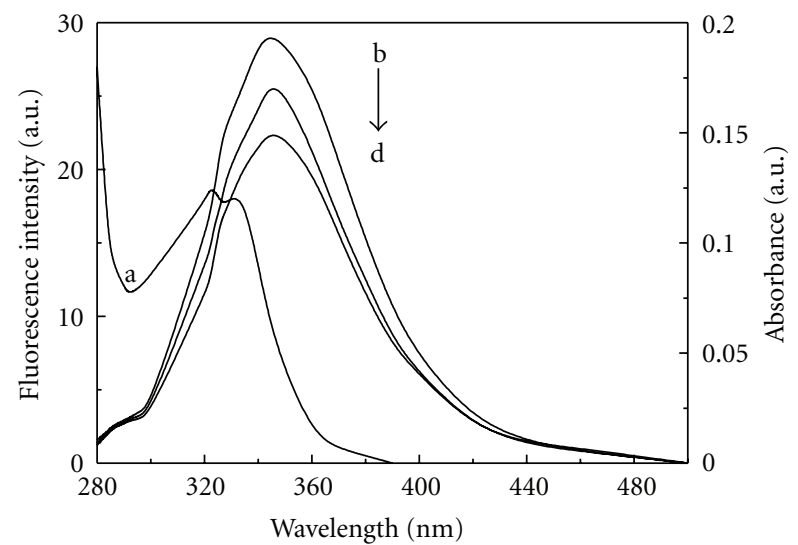

FIGURE 5: Fluorescence spectra for BSA and BSA-SY and absorbance spectra for $\operatorname{CPFX}(T=298 \mathrm{~K})$. $C_{\mathrm{BSA}}=C_{\mathrm{CPFX}}=10.0 \mu \mathrm{M}$ in all systems; a: absorption of CPFX; b: fluorescence of BSA; c: fluorescence of BSA-SY, $C_{\mathrm{SY}}=2.0 \mu \mathrm{M}$; d: fluorescence of BSA-SY, $C_{\mathrm{SY}}=5.0 \mu \mathrm{M}$.

spectra and difference spectra of BSA-CPFX system in the presence of synthetic food colorants (such as SY) are illustrated in Figure 4. We studied effects of TTZ, SY, and ETS on the binding constants between CPFX and BSA according to (3). The $\varphi_{2}$ represents the rate of change of binding constants with synthetic food colorants added in BSA-CPFX system. It was determined using

$$
\varphi_{2}=\frac{\left(K_{a}^{\prime \prime}-K_{a(\mathrm{BSA}-\mathrm{CPFX})}\right)}{K_{a(\mathrm{BSA}-\mathrm{CPFX})}},
$$

where $K_{a}^{\prime \prime}$ is the binding constant of the BSA-CPFX system in the presence of synthetic food colorants. Table 3 gives the corresponding calculated results. The negative $\varphi_{2}$ values showed the synthetic food colorants made the formation of BSA-CPFX more difficult. This was attributed to the site I for food colorants and CPFX on BSA. The food colorants could compete with CPFX for the binding sites from BSA. Meanwhile, the combination abilities of these synthetic food colorants with BSA were stronger than CPFX with BSA. Probably, food colorants induced the release or displacement of CPFX from its binding sites in BSA, and hence the free, biological active fraction of CPFX increased. The values of $\varphi_{2}$ increased in following order: ETS $<$ TTZ $<$ SY, suggesting the influences of food colorants on the BSA-CPFX complex followed the pattern: ETS $>$ TTZ $>$ SY. These results indicated that the increase of the combination ability of food colorants to BSA might enhance the competition with BSA and deepen the influences of food colorants on the formation of BSA-CPFX complex. The values of $\varphi_{2}$ were much smaller with the increase in the concentration of food colorants. It showed that the competition of food colorants and CPFX was enhanced and the free fraction of CPFX further increased. In addition, all systems had a good linearity. The presence of the synthetic food colorants did not affect the number of binding site of BSA-CPFX system, which was still about 1.

3.5. Binding Distances between the Drugs and BSA in the Absence and Presence of Synthetic Food Colorants. The overlap of UV-vis absorption spectra of CPFX with the fluorescence emission spectra of BSA and BSA-synthetic food colorants (such as SY) were shown in Figure 5. According to 
TABLE 3: Binding constants $K_{a}^{\prime \prime}$, number of binding sites $n$ and the rate of change of binding constants $\varphi_{2}$ for BSA-CPFX system in the presence of food colorants at $298 \mathrm{~K}$.

\begin{tabular}{|c|c|c|c|c|c|}
\hline System & $C_{\text {colorants }}(\mu \mathrm{M})$ & $K_{a}^{\prime \prime}\left(\mathrm{M}^{-1}\right)$ & $n$ & $R^{\prime b}$ & $\varphi_{2}(\%)$ \\
\hline \multirow{2}{*}{ BSA-ETS-CPFX } & 2.0 & $2.77 \times 10^{4}$ & 1.25 & 0.9988 & -8.28 \\
\hline & 5.0 & $2.12 \times 10^{4}$ & 0.984 & 0.9991 & -29.8 \\
\hline \multirow{2}{*}{ BSA-TTZ-CPFX } & 2.0 & $2.87 \times 10^{4}$ & 1.06 & 0.9972 & -4.97 \\
\hline & 5.0 & $2.39 \times 10^{4}$ & 1.11 & 0.9912 & -20.9 \\
\hline \multirow{2}{*}{ BSA-SY-CPFX } & 2.0 & $2.91 \times 10^{4}$ & 1.11 & 0.9984 & -3.96 \\
\hline & 5.0 & $2.53 \times 10^{4}$ & 1.01 & 0.9958 & -16.2 \\
\hline
\end{tabular}

$\overline{R^{\prime} \mathrm{b}}$ The linear relative coefficient of $\lg \left[\left(F_{0}-F\right) / F\right] \sim \lg \left\{\left[Q_{t}\right]-n\left[B_{t}\right]\left(F_{0}-F\right) / F_{0}\right\}$.

TABLE 4: Energy transfer efficiency $(E)$ and parameters $J, r, R_{0}$ for systems.

\begin{tabular}{|c|c|c|c|c|c|}
\hline System & $C_{\text {colorants }}(\mu \mathrm{M})$ & $E(\%)$ & $J\left(\mathrm{~cm}^{3} \mathrm{M}^{-1}\right)$ & $R_{0}(\mathrm{~nm})$ & $r(\mathrm{~nm})$ \\
\hline BSA-TTZ & - & 29.5 & $1.127 \times 10^{-14}$ & 2.50 & 2.89 \\
\hline BSA-SY & - & 23.5 & $5.181 \times 10^{-15}$ & 2.20 & 2.67 \\
\hline BSA-ETS & - & 11.0 & $4.536 \times 10^{-16}$ & 1.46 & 2.07 \\
\hline BSA-CPFX & 0 & 23.6 & $7.43 \times 10^{-15}$ & 2.33 & 2.83 \\
\hline \multirow{2}{*}{ BSA-TTZ-CPFX } & 2.0 & 22.2 & $7.44 \times 10^{-15}$ & 2.33 & 2.87 \\
\hline & 5.0 & 21.3 & $7.51 \times 10^{-15}$ & 2.34 & 2.91 \\
\hline \multirow{2}{*}{ BSA-SY-CPFX } & 2.0 & 23.3 & $7.43 \times 10^{-15}$ & 2.33 & 2.84 \\
\hline & 5.0 & 22.6 & $7.36 \times 10^{-15}$ & 2.33 & 2.86 \\
\hline \multirow{2}{*}{ BSA-ETS-CPFX } & 2.0 & 17.7 & $7.41 \times 10^{-15}$ & 2.33 & 3.01 \\
\hline & 5.0 & 17.3 & $7.44 \times 10^{-15}$ & 2.33 & 3.02 \\
\hline
\end{tabular}

Förster's theory, the energy donor and the energy acceptor distance $r$ is related with the critical energy transfer distance $R_{0}$ and energy efficiency $E$ can be calculated by the formula [22]:

$$
\begin{gathered}
E=1-\frac{F}{F_{0}}=\frac{R_{0}^{6}}{\left(r^{6}+R_{0}^{6}\right)}, \\
R_{0}^{6}=8.78 \times 10^{-25} K^{2} \Phi N^{-4} J,
\end{gathered}
$$

where $K^{2}$ is the orientation factor, $\Phi$ is the fluorescence quantum yield of the donor, $N$ is a refractive index of the medium, $J$ is the overlap integral between the fluorescence emission spectrum of the donor and the absorption spectrum of the acceptor, and $J$ is given by [23]

$$
J=\frac{\sum F(\lambda) \varepsilon(\lambda) \lambda^{4} \Delta \lambda}{\sum F(\lambda) \Delta \lambda},
$$

where $F(\lambda)$ is the fluorescence intensity of the fluorescence donor at wavelength $\lambda$ and $\varepsilon(\lambda)$ is the molar absorption coefficient of the acceptor at this wavelength. Under these experimental conditions, it has been reported that $K^{2}=2 / 3$, $N=1.336$, and $\Phi=0.118$. Thus, $J, E, R_{0}$, and $r$ were calculated as shown in Table 4 .

The fluorescence of BSA comes from the tryptophan residue 212 in subdomain IIA [24], so $r$ is the distance between binding sites and this residue. Tryptophan residue is situated in the hydrophobic cavity of BSA, thus fat-soluble drugs have an easier access to the cavity to form complexes with BSA. CPFX is water soluble and difficult to enter the cavity and the binding position is relatively far from this tryptophan. So the values $r$ are greater than the hydrophobic drug of Ligustrazine Hydrochloride $(r=1.8 \mathrm{~nm})$. It appeared that the binding distances decreased in following order: $r_{\text {(BSA-ETS-CPFX) }}>r_{(\mathrm{BSA}-\mathrm{TTZ}-\mathrm{CPFX})}>r_{\text {(BSA-SY-CPFX) }}>r_{\text {(BSA-CPFX) }}$. Furthermore, with the increase of concentration of colorants, the values of $r$ increased. These results indicated that the binding stability of BSA-CPFX complex decreased in the presence of synthetic food colorants.

\section{Conclusions}

Coadministration of two ligands may influence each other's binding with protein. The binding constant of BSA-CPFX system was decreased, and the binding distance was increased in the presence of synthetic food colorants TTZ, SY, and ETS. Hence, the free fraction of CPFX was increased, and the efficacy of CPFX would be affected. Consequently, to avoid the competition between food colorants and drugs, food containing synthetic colorants should be minimized or forbidden during application of CPFX. This paper studied only the influences of three widely used synthetic colorants TTZ, SY, and ETS on the efficacy of a common quinolones CPFX. However, the influences of other pigments and those illegally added pigments such as sudan red and bromocresol green on the drugs, which contribute to common diseases like hyperlipemia, hyperlipidemia, cancer, and diabetes, were not proved, and therefore, further research is expected. 


\section{Acknowledgments}

The authors gratefully acknowledge the financial support of National Science Foundation of China (Grant no. 20675024) and Hebei Provincial Key Basic Research Program (Grant no. 10967126D).

\section{References}

[1] C. Wang, Q. H. Wu, Z. Wang, and J. Zhao, "Study of the interaction of carbamazepine with bovine serum albumin by fluorescence quenching method," Analytical Sciences, vol. 22, no. 3, pp. 435-438, 2006.

[2] A. Sułkowska, M. Maciazek-Jurczyk, B. Bojko et al., "Competitive binding of phenylbutazone and colchicine to serum albumin in multidrug therapy: a spectroscopic study," Journal of Molecular Structure, vol. 881, no. 1-3, pp. 97-106, 2008.

[3] N. Shahabadi and M. Maghsudi, "Binding studies of a new copper (II) complex containing mixed aliphatic and aromatic dinitrogen ligands with bovine serum albumin using different instrumental methods," Journal of Molecular Structure, vol. 929, no. 1-3, pp. 193-199, 2009.

[4] X. L. Han, P. Mei, Y. Liu, Q. Xiao, F. L. Jiang, and R. $\mathrm{Li}$, "Binding interaction of quinclorac with bovine serum albumin: a biophysical study," Spectrochimica Acta Part A, vol. 74, no. 3, pp. 781-787, 2009.

[5] B. Bojko, A. Sułkowska, M. Maciazek-Jurczyk, J. Równicka, and W. W. Sułkowski, "The influence of dietary habits and pathological conditions on the binding of theophylline to serum albumin," Journal of Pharmaceutical and Biomedical Analysis, vol. 52, no. 3, pp. 384-390, 2010.

[6] A. M. Evans, "Influence of dietary components on the gastrointestinal metabolism and transport of drugs," Therapeutic Drug Monitoring, vol. 22, no. 1, pp. 131-136, 2000.

[7] L. W. Zhang, K. Wang, and X. X. Zhang, "Study of the interactions between fluoroquinolones and human serum albumin by affinity capillary electrophoresis and fluorescence method," Analytica Chimica Acta, vol. 603, no. 1, pp. 101-110, 2007.

[8] M. Guo, W. J. Lu, P. G. Yi, and Q. S. Yu, "Study on the thermodynamic characteristics between fluoroquinolone and bovine serum albumin," Journal of Chemical Thermodynamics, vol. 39, no. 3, pp. 337-343, 2007.

[9] M. Ma, X. Luo, B. Chen, S. Su, and S. Yao, "Simultaneous determination of water-soluble and fat-soluble synthetic colorants in foodstuff by high-performance liquid chromatography-diode array detection-electrospray mass spectrometry," Journal of Chromatography A, vol. 1103, no. 1, pp. 170-176, 2006.

[10] G. Lupidi, A. Scire, E. Camaioni et al., "Thymoquinone, a potential therapeutic agent of Nigella sativa, binds to site I of human serum albumin," Phytomedicine, vol. 17, no. 10, pp. 714-720, 2010.

[11] F. Ding, G. Zhao, S. Chen, F. Liu, Y. Sun, and L. Zhang, "Chloramphenicol binding to human serum albumin: determination of binding constants and binding sites by steadystate fluorescence," Journal of Molecular Structure, vol. 929, no. 1-3, pp. 159-166, 2009.

[12] I. Matei and M. Hillebrand, "Interaction of kaempferol with human serum albumin: a fluorescence and circular dichroism study," Journal of Pharmaceutical and Biomedical Analysis, vol. 51, no. 3, pp. 768-773, 2010.
[13] Y. Q. Wang, H. M. Zhang, and Q. H. Zhou, "Investigation of the interaction between pentachlorophenol and human serum albumin using spectral methods," Journal of Molecular Structure, vol. 932, no. 1-3, pp. 31-37, 2009.

[14] J. B. Xiao, X. Q. Chen, X. Y. Jiang, M. Hilczer, and M. Tachiya, "Probing the interaction of trans-resveratrol with bovine serum albumin: a fluorescence quenching study with tachiya model," Journal of Fluorescence, vol. 18, no. 3-4, pp. 671-678, 2008.

[15] Y. L. Wei, J. Q. Li, C. Dong, S. M. Shuang, D. S. Liu, and C. W. Huie, "Investigation of the association behaviors between biliverdin and bovine serum albumin by fluorescence spectroscopy," Talanta, vol. 70, no. 2, pp. 377-382, 2006.

[16] A. Asadi, A. A. Saboury, A. A. Moosavi-Movahedi, A. Divsalar, and M. N. Sarbolouki, "Interaction of bovine serum albumin with some novel PEG-containing diblock copolymers," International Journal of Biological Macromolecules, vol. 43, no. 3, pp. 262-270, 2008.

[17] E. L. Gelamo and M. Tabak, "Spectroscopic studies on the interaction of bovine (BSA) and human (HSA) serum albumins with ionic surfactants," Spectrochimica Acta Part A, vol. 56, no. 11, pp. 2255-2271, 2000.

[18] F. Cui, Y. Cui, H. Luo, X. Yao, J. Fan, and Y. Lu, "Interaction of APT with BSA or HSA," Chinese Science Bulletin, vol. 51, no. 18, pp. 2201-2207, 2006.

[19] K. Yamasaki, T. Maruyama, U. Kragh-Hansen, and M. Otagiri, "Characterization of site I on human serum albumin: concept about the structure of a drug binding site," Biochimica et Biophysica Acta, vol. 1295, no. 2, pp. 147-157, 1996.

[20] J. Tian, J. Liu, X. Tian, Z. Hu, and X. Chen, "Study of the interaction of kaempferol with bovine serum albumin," Journal of Molecular Structure, vol. 691, no. 1-3, pp. 197-202, 2004.

[21] S. Ercelen, A. S. Klymchenko, Y. Mély, and A. P. Demchenko, "The binding of novel two-color fluorescence probe FA to serum albumins of different species," International Journal of Biological Macromolecules, vol. 35, no. 5, pp. 231-242, 2005.

[22] M. Gharagozlou and D. M. Boghaei, "Interaction of watersoluble amino acid Schiff base complexes with bovine serum albumin: fluorescence and circular dichroism studies," Spectrochimica Acta Part A, vol. 71, no. 4, pp. 1617-1622, 2008.

[23] S. N. Khan, B. Islam, R. Yennamalli, A. Sultan, N. Subbarao, and A. U. Khan, "Interaction of mitoxantrone with human serum albumin: spectroscopic and molecular modeling studies," European Journal of Pharmaceutical Sciences, vol. 35, no. 5, pp. 371-382, 2008.

[24] B. Bojko, A. Sułkowska, M. Maciazek-Jurczyk, J. Równicka, and W. W. Sułkowski, "Investigations of acetaminophen binding to bovine serum albumin in the presence of fatty acid: fluorescence and 1H NMR studies," Journal of Molecular Structure, vol. 924-926, pp. 332-337, 2009. 

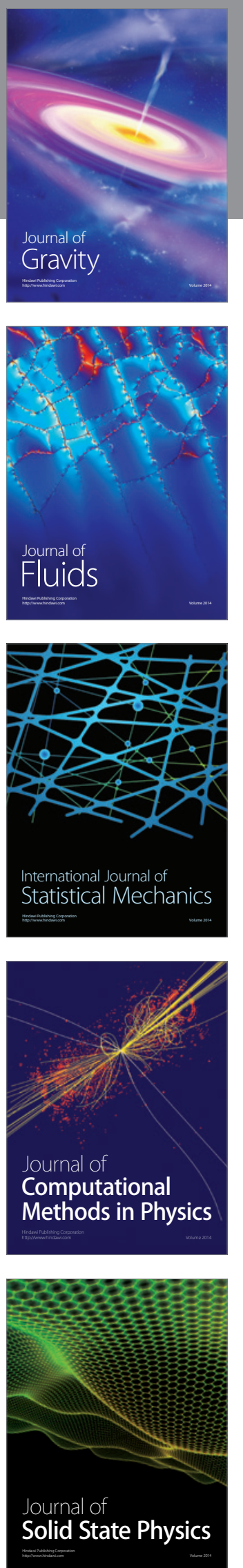

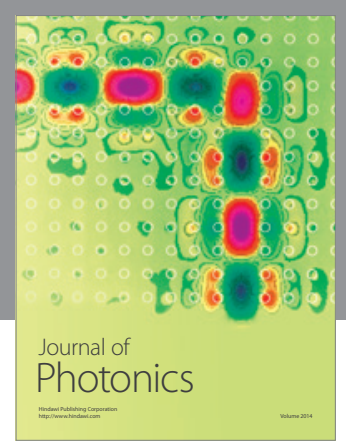

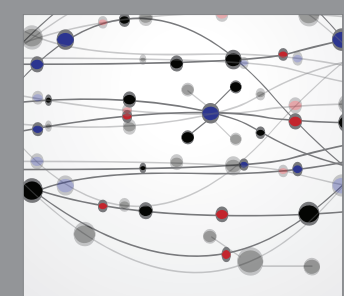

The Scientific World Journal
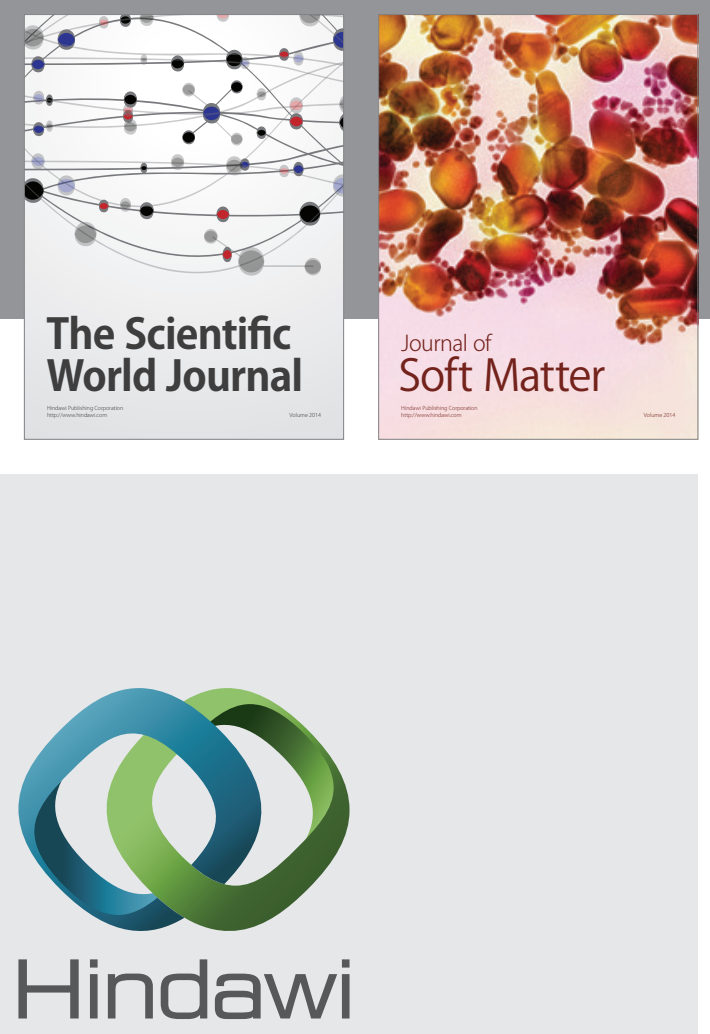

Submit your manuscripts at

http://www.hindawi.com
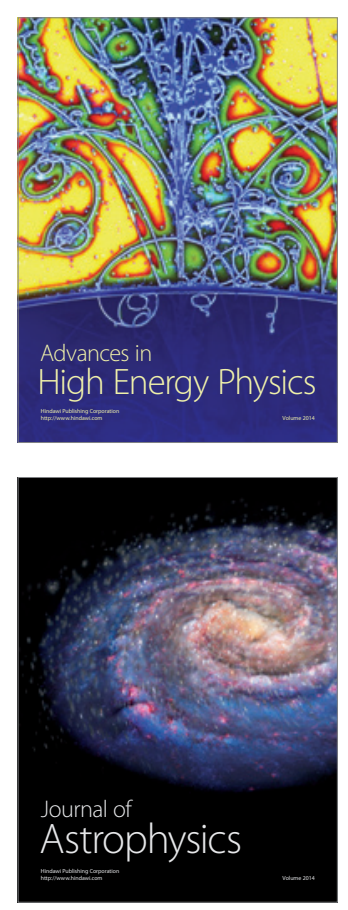
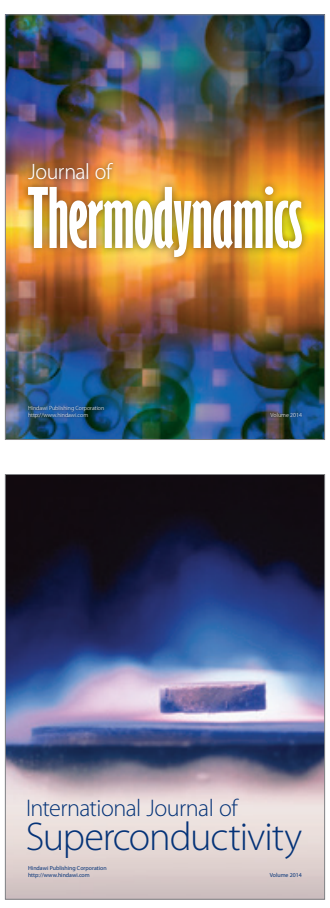
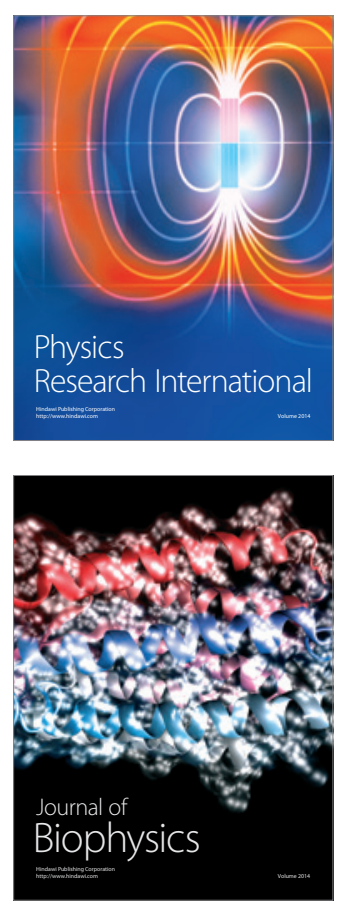
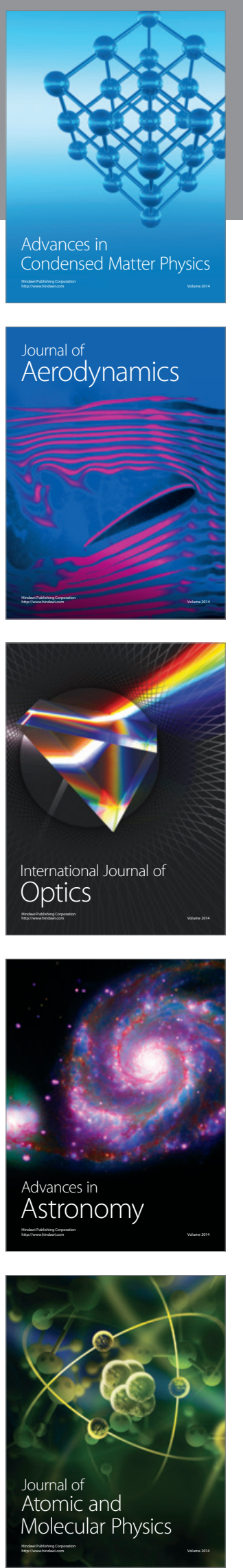